\title{
Bronchial Injury
}

National Cancer Institute

\section{Source}

National Cancer Institute. Bronchial Injury. NCI Thesaurus. Code C35453.

Trauma to the lung bronchi. 